\title{
Mindfulness and yoga halt the conversion of pre-diabetic rural women into diabetics-a pilot study
}

\author{
Navneet Kaur ${ }^{1,6}$, Neeru Malik², Deepali Mathur ${ }^{3}$, Surinder Pal ${ }^{4}$, Rakesh Malik ${ }^{5}$, Sumit Rana ${ }^{6}$ \\ ${ }^{1}$ Department of Physical Education, Panjab University, Chandigarh, India \\ ${ }^{2}$ Dev Samaj College of Education, Sector 36B, Chandigarh, India \\ ${ }^{3}$ School of Biotechnology, KIIT University, Bhubaneshwar, Odisha, India \\ ${ }^{4}$ Centre for Systems Biology and Bioinformatics, Panjab University, Chandigarh, India \\ ${ }^{5}$ Department of Physical Education and Sports, Panjab University, Chandigarh, India \\ ${ }^{6}$ Neuroscience Research Lab, Department of Neurology, Postgraduate Institute of Medical Education and Research, Chandigarh, India
}

\section{KEY WORDS}

Glycated Haemoglobin Diabetic Yoga Protocol Indian Diabetes Risk Score Prediabetes

\section{${ }^{*}$ Corresponding Author:}

Neeru Malik, PhD

Dev Samaj College of Education

Sector 36B, Chandigarh

Contact no: 9781133666

E-mail: drneerumalikhry@gmail.com

\begin{abstract}
Background and Purpose: A plethora of studies have been conducted in the past, which have proven the salutary effects of yoga practice. Yoga and other mind body techniques are deemed as stress relievers and have shown therapeutic effectiveness in preventing the development and progression of several lifestyle diseases including Diabetes. Yoga practice may help in managing Diabetes by regulating the glucose levels in blood. In this study we have evaluated the efficacy of three months of AYUSH-approved Diabetic Yoga Protocol (DYP) on high-risk individuals. Methods: Thirty three high-risk female participants with Indian Diabetes Risk Score (IDRS) $\geq 60$ was recruited for a period of three months. The participants were divided into Diabetic Yoga Protocol Group ( $\mathrm{N}=18$ ) and Control Group ( $\mathrm{N}=15)$. Alteration for selected glycemic, psychological, and anthropometric parameter of individuals was examined at the baseline and after three months of Yoga practices.

Results: The three months of Yoga practice demonstrated significant changes on the levels of $\operatorname{HbA1c}(p=0.010)$, Body Mass Index (BMI) $(p=0.006)$, sustained attention $(p=0.012)$, general health $(p=0.002)$, state anxiety $(p=0.001)$, perceived stress $(p=0.004)$, IDRS $(p<0.001)$ and overall Quality of life $(p<0.001)$. However, no significant changes were seen for Fasting Blood Sugar (FBS), and Waist Hip Ratio (WHR). While, in the control group, IDRS ( $p=0.019)$ was significantly increased, significant decrease in BMI $(p=0.011)$ and state anxiety $(p=0.016)$ was observed. However, no significant changes were seen in HbA1c, FBS, WHR, Sustained Attention, General Health, Perceived Stress and Overall QOL.

Conclusion: The findings of the present study are suggestive of DYP as a possible application for public health intervention regime for women.
\end{abstract}

doi: 10.38205/imcr.010207

\section{Introduction}

Diabetes is a chronic epidemic whose prevalence is increasing across the globe causing a major economic burden on the society $(1,2)$. According to the International Diabetes Federation, 72.9 million people have been reported to suffer from Diabetes in India (3). It is the seventh principal cause of death as per WHO estimates. There are several micro-and macrovascular complications known to be linked with Diabetes and, therefore, timely detection of the disease at prediabetic stage might help prevent its onset (4).

In the modern scenario, lifestyle risk factors like obesity, sedentary lifestyle, urbanization, poor eating habits are the most prevalent causes for the onset of Diabetes and Prediabetes (5). Prediabetic condition is a state which occurs prior to the onset of Diabetes $(6,7)$ and can be treated if appropriate measures are taken. Impaired fasting glucose (IFG) and impaired glucose tolerance (IGT) are its other forms (8). Risk factors including parental history, physical inactivity (9), age etc. are all considered as contributors in the development and progression of Diabetes. Safford et al. (2005) revealed that lack of sleep, anxiety, depression and stress are the main culprits in Asian Population (10). To detect the undiagnosed Diabetes and high risk individuals in Indian population, Mohan et al. (2005) developed the "Indian Diabetes Risk Score" (IDRS), which is a well-established method based on four simple criteria: a) age, b) level of physical activity, c) family history and d) waist circumference (11). Studies suggest that inactive lifestyle and poor diet are the modifiable risk factors in prediabetic people $(12,13)$. In the current post-modern living, there is need for non-pharmacological lifestyle modification tools to prevent Diabetes and other diseases (14). Previous reports indicate that adopting a healthier lifestyle along with standard treatment (15) may diminish diabetic complications and may also decrease the risk rate of Diabetes by 63\%-65\% in prediabetics (16). In addition, there is a $58 \%$ reduction in risk of conversion of Diabetics through lifestyle intervention, which focus upon weight loss, and enhancement of physical activity (17). 
Yoga is an ancient Indian discipline, which is focused upon synchronization of mind, body and soul. The practice of Yoga comprises of various pranayama (breathing practices), asanas (Physical postures) and Dhayana (meditation) (18). It is based on the wholesome treatment of the individual, which focuses on a healthy body free of toxins, purification of mind and emotional well-being. In Yogic philosophy, it is believed that the cause of disease is not only physical but also its mental and emotional aspects may play an equally significant role. Therefore, for management of ailments, treatment strategy must address both physical and mental aspects alike (19).

Furthermore, Yoga has been found to have a positive impact on pre-diabetic individuals especially regarding risk reduction of Diabetes along with reducing the cardiometabolic risk. (20). There are some studies which have reported other lifestyle interventions like dietary change and inclusion of physical activity that aid weight loss and delay the conversion from prediabetics into diabetes (21-23) but the role of Yoga in enabling the same has not been overemphasized. Several lines of evidence have shown that lifestyle modification measures are protective and a cost-effective method to prevent and manage the disease $(22,24-26)$.

In the changing scenario, the Diabetes severely affected the rural areas in India with expected increasing trend from $1 \%$ to $4-10 \%$ annually. The increasing prevalence of Diabetes in the rural areas is a matter of concern as the major part (72.2\%) of Indian population resides in the rural regions (27). Hence, the timely preventive strategy like Yoga might helps in delaying the onset of Diabetes in rural population. In the present study, we wanted to examine the biochemical, anthropometric and psychological effects of DYP on high-risk individuals in order to understand the impact of Yoga as a promising preventive strategy for prediabetic individuals in rural Indian women.

\section{Methods}

\section{Recruitments}

A door to door screening was carried out for the identification of high-risk individuals among the rural population of Chandigarh (U.T) based on IDRS. Furthermore, written informed consents were taken from every participant during door to door screening as well as at the time of registration. The study protocol was approved by ethics committee of Panjab University Institutional Ethics Committee (PUIEC) vide letter no. PUIEC/2017/80/A-1/08/08.

Out of 71 screened participants those with less than IDRS 60 score $(\mathrm{N}=34$ ) were excluded from the study. The high risk individuals with IDRS score $\geq 60$ were included in the study and to the remaining 37 subjects were further divided into two equal groups i.e Yoga Group ( $N=19)$ and Control Group ( $\mathrm{N}=18$ ) assessed for Biochemical (FBS \& HbA1c), Psychological (Attention, State anxiety \& Perceived Stress), and Anthropometric (BMI \& WHR) parameters. Moreover, General Health and Quality of life of the participants was also measured. Initially, 37 participants were recruited in the study. At the end, there were 33 participants with one dropout in Yoga group and 3 dropouts in control group. The major exclusion criteria in the study was known Diabetics, or any major disease or illness. Figure 1 shows the schematic presentation of the study participants.

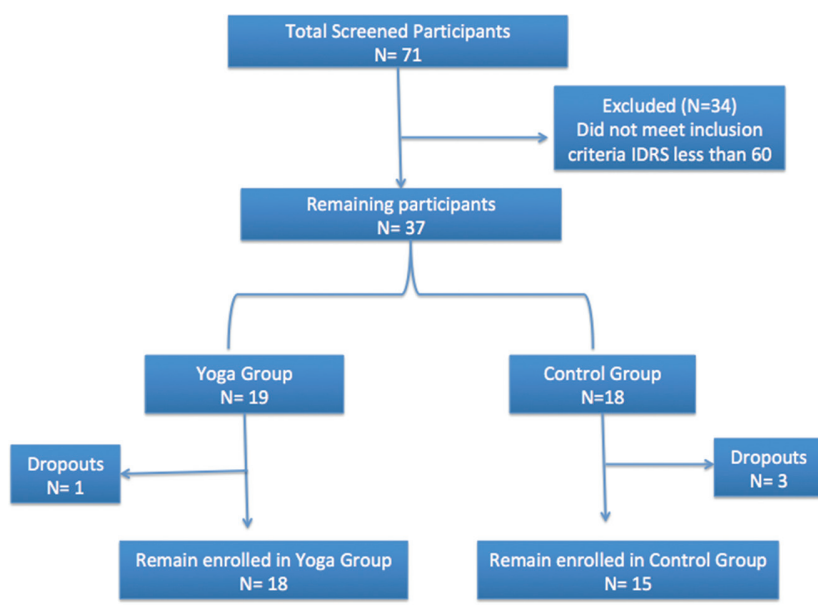

Fig. 1: Schematic representation of the study participants.

\section{Measures}

The study population consisted of healthy individuals $(n=14)$, Pre-diabetics $(n=15)$ and Diabetics $(n=4)$ cases. The assessments viz: Body Mass Index (BMI), Waist Hip Ratio (WHR), Fasting Blood Glucose, HbA1c, Perceived stress scale (PSS), State and Trait Anxiety (STAI,) General Health, Quality of Life (QOL), Sustained attention were carried out at Baseline and after 3 months. Socio-demographic characteristics of the study participants are provided in Table 1.

Table 1: Sociodemographic characteristics of the study participants

\begin{tabular}{|l|c|}
\hline Socio-Demographic Characteristics $(\mathrm{n}=25)$ & Values \\
\hline Age (Years) & $42.27 \pm 8.18$ \\
\hline Gender (Female Only) & 38 \\
\hline Yoga Group & 19 \\
Dropouts & 1 \\
Control Group & 18 \\
Dropouts & 3 \\
\hline Previous Yoga Experience & Yes-0 \\
& No-33 \\
\hline Marital Status & Yes-33 \\
& No-0 \\
\hline Education Level & \\
Illiterate & 6 \\
Primary School & 6 \\
Middle School & 1 \\
High School & 10 \\
Senior Secondary School & 5 \\
Graduate & 3 \\
Post Graduate & 2 \\
\hline Occupation & \\
Household & 27 \\
Self Employed & 2 \\
Working & 4 \\
\hline
\end{tabular}


The biochemical assessments such as FBS and $\mathrm{HbA1c} \%$ were measured after collection of blood sample by a diagnostic laboratory using standard diagnostic procedures. Fasting blood sugar (FBS) (Rxl-Max 500) was measured in early morning after 10-12 hrs of fasting and the level of A1c (Bio-Rad D-10) was also estimated.

The BMI and WHR of the participants was calculated by using the formulae: $\mathrm{BMI}=$ weight (in Kilograms)/height (in meters) ${ }^{2}$ and $(\mathrm{W} / \mathrm{H})$ respectively.

The attention level of the participants was assessed by six letter cancellation test (SLCT) constructed by Natu and Agarwal (1997). This task consisted of 22 columns and 14 rows of random letters and the participants were required to cancel only the six-letter mentioned in the sheet within the 90 seconds. The net attempted score was obtained by subtracting the wrongly attempted letters from the total attempted letters. (28)

The State anxiety of the participants was assessed by using the sub-scale of state and trait anxiety inventory constructed by Spielberger (1983). This sub-scale comprised of 20 questions. The reverse scoring was done for 10 items. (29) Moreover, the perceived stress of the participants was evaluated by Perceived Stress Scale (PSS) made by Cohen, Kamarck, Mermelstein (1994). This questionnaire used consists of 10 items and reversed scoring was done for 4 items. (30)

The General health was analysed by using General Health Questionnaire -12 (GHQ-12). The scale contained 12 items and measures and the level of psychological distress in the participants (31). The Quality of Life of the participants was assessed using the by BREF-WHOQOL scale. The scale consisted of a total of 26 items which further divided into four domains i.e. physical domain, social domain, psychological domain and environmental domain. The reverse scoring was done for three items. (32)

\section{Interventions}

A Yoga Centre was identified for this study in the rural village. The DYP which is especially designed for prediabetics and diabetics was administered in this study for a total duration of 60 minutes. The Yogic practices included: Starting prayer, loosening exercises, Suryannamskara and asanas, Pranayam, and meditation. The entire protocol was published previously (33). The Yoga session was administered or taught by qualified Yoga instructor(s).

\section{Statistical analysis}

Statistical package for social sciences (SPSS), version 22.0. was used for statistical analysis. First, the normality of the data was tested or quantified by using the Kolmogorov-Smirnov test. The data was found to be normally distributed for all the variables $(p>0.05)$. The paired t-test was used to find out the differences and changes at baseline and after three months yoga practice. $p$ value along with mean, SD and CI was also calculated. $\mathrm{p}$ value $<0.05$ was considered statistically significant.

\section{Results}

The baseline characteristics of the participants in both Yoga and Control group are shown in Table 2. Our findings revealed significant improvement in glycated haemoglobin $(\mathrm{p}=0.010)$, BMI $(p=0.006)$, IDRS $(p<0.001)$, SLCT $(p=0.012)$, general health $(p=0.002)$, state anxiety $(p<0.001)$, PSS $(p=0.004)$, social $(p=0.006)$, and psychological ( $p=0.026)$, domains of QOL and overall QOL ( $\mathrm{p}<0.001)$ in DYP group after three months Yoga practice in high risk individuals as shown in Table 3 and Figure 2. However, no statistically significant changes were seen for Fasting blood sugar, Waist hip ratio (WHR), Physical and environmental domain of QOL as Shown in Figure 2 and Table 3.

In control group the finding shows significant increase in IDRS ( $p=0.019)$, and significant decline in BMI ( $p=0.011)$, STAI ( $p=0.016)$ after three months as shown in Figure 3 and Table 3. However, no significant changes were observed in Hba1c, FBS, WHR, Attention, PSS, Physical, Psychological, Social, Environmental domain and Overall QOL as shown in Figure 3 and Table 3. Moreover, in DYP group more people were found converted into healthy individuals $(44.44 \%$ to $72.22 \%$ ). Similarly, in the Prediabetic group the proportion of Prediabetics dropped (44.44\% to $16.67 \%$ ) after threemonths as shown in Figure 4 (a) in comparison with control group as shown in Figure 4 (b). In both the groups no changes was observed in Diabetic group.

Table 2: Baseline characteristics of the participants in the DYP and Control group

\begin{tabular}{|l|c|c|}
\hline Characteristic & $\begin{array}{c}\text { Yoga group } \\
\mathrm{n}=18\end{array}$ & $\begin{array}{c}\text { Control group } \\
\mathrm{n}=15\end{array}$ \\
\hline IDRS & $71.67 \pm 10.43$ & $70.67 \pm 10.33$ \\
\hline Age (years) & $40.55 \pm 7.94$ & $44.33 \pm 8.25$ \\
\hline BMI & $30.45 \pm 5.60$ & $30.80 \pm 3.90$ \\
\hline WHR & $0.89 \pm 0.068$ & $0.90 \pm 0.063$ \\
\hline FBS & $86.52 \pm 13.56$ & $91.45 \pm 22.95$ \\
\hline HbA1c & $5.77 \pm 0.48$ & $5.9 \pm 0.57$ \\
\hline SLCT & $20.89 \pm 9.76$ & $19.93 \pm 9.82$ \\
\hline GHQ & $13.94 \pm 4.30$ & $12.53 \pm 4.08$ \\
\hline STAI & $44.61 \pm 10.27$ & $46.33 \pm 9.66$ \\
\hline PSS & $18.72 \pm 5.42$ & $21 \pm 3.53$ \\
\hline PHYSICAL & $54.28 \pm 9.91$ & $58.87 \pm 7.13$ \\
\hline PSYCHOLOGICAL & $50.77 \pm 9.33$ & $55.47 \pm 9.16$ \\
\hline SOCIAL & $59.67 \pm 12.61$ & $63.33 \pm 15.40$ \\
\hline ENVIRONMENTAL & $72.11 \pm 8.72$ & $70.6 \pm 9.40$ \\
\hline OVERALL QOL & $236.83 \pm 18.33$ & $246.6 \pm 27.23$ \\
\hline
\end{tabular}


Table 3: Table showing changes in selected Biochemical, Anthropometric, Psychological and General wellbeing assessments in Mean, SD and SEM values at baseline and after 3 months in DYP and Control Group

\begin{tabular}{|l|c|c|c|c|c|c|}
\hline & \multicolumn{3}{|c|}{ DYP Group } & \multicolumn{3}{c|}{ Control Group } \\
\hline Variables & $\begin{array}{c}\text { Baseline } \\
\text { Mean } \pm \text { SD }) \\
(95 \% \text { CI })\end{array}$ & 3 Months & P Value & $\begin{array}{c}\text { Baseline } \\
\text { (Mean } \pm \text { SD }) \\
95 \% \text { CI })\end{array}$ & 3 Months & P Value \\
\hline HbA1c & $5.77 \pm .48$ & $5.58 \pm .52$ & $\mathbf{0 . 0 1 0}$ & $5.90 \pm .57$ & $5.70 \pm .468$ & 0.175 \\
\hline FBS & $86.52 \pm 13.56$ & $81.52 \pm 13.41$ & 0.231 & $91.44 \pm 22.95$ & $92.78 \pm 23.69$ & 0.758 \\
\hline BMI & $30.45 \pm 5.60$ & $29.66 \pm 5.42$ & $\mathbf{0 . 0 0 6}$ & $30.80 \pm 3.90$ & $30.18 \pm 3.70$ & $\mathbf{0 . 0 1 1}$ \\
\hline WHR & $.89 \pm .068$ & $.90 \pm .0528$ & 0.313 & $.90 \pm .063$ & $.88 \pm .0531$ & 0.277 \\
\hline SLCT & $20.89 \pm 9.76$ & $27.89 \pm 13.01$ & $\mathbf{0 . 0 1 2}$ & $19.93 \pm 9.82$ & $20.13 \pm 11.41$ & 0.909 \\
\hline GHQ & $13.94 \pm 4.30$ & $8.56 \pm 4.36$ & $\mathbf{0 . 0 0 2}$ & $12.53 \pm 4.085$ & $12.67 \pm 2.34$ & 0.690 \\
\hline PSS & $18.72 \pm 5.42$ & $13.78 \pm 3.32$ & $\mathbf{0 . 0 0 4}$ & $21.00 \pm 3.53$ & $18.20 \pm 5.21$ & 0.052 \\
\hline STAI & $44.61 \pm 10.27$ & $32.00 \pm 11.57$ & $\mathbf{0 . 0 0 1}$ & $46.33 \pm 9.66$ & $38.00 \pm 6.98$ & $\mathbf{0 . 0 1 6}$ \\
\hline IDRS & $71.67 \pm 10.43$ & $64.44 \pm 13.38$ & $<\mathbf{0 . 0 0 1}$ & $70.67 \pm 10.327$ & $74.00 \pm 11.21$ & $\mathbf{0 . 0 1 9}$ \\
\hline Physical Domain & $54.28 \pm 9.91$ & $58.17 \pm 9.60$ & 0.116 & $58.87 \pm 7.13$ & $56.40 \pm 8.55$ & 0.237 \\
\hline Social Domain & $59.67 \pm 12.61$ & $72.94 \pm 13.20$ & $\mathbf{0 . 0 0 6}$ & $63.33 \pm 15.40$ & $65.80 \pm 12.44$ & 0.510 \\
\hline Psycho Domain & $50.78 \pm 9.33$ & $58.72 \pm 9.07$ & $\mathbf{0 . 0 2 6}$ & $55.47 \pm 9.16$ & $57.06 \pm 6.73$ & 0.543 \\
\hline Environmental Domain & $72.11 \pm 8.72$ & $73.33 \pm 9.54$ & 0.677 & $70.60 \pm 9.40$ & $71.00 \pm 10.52$ & 0.867 \\
\hline QOL & $236.83 \pm 18.33$ & $263.17 \pm 30.08$ & $<\mathbf{0 . 0 0 1}$ & $246.60 \pm 27.22$ & $250.27 \pm 29.58$ & 0.543 \\
\hline
\end{tabular}

Data Expressed in Mean, SD, SEM and Statistical significance $\left(p<.001^{* * *}, p=0.001^{* *}, p=0.002^{* *}, p=0.004^{* *}, p=0.006^{* *}, p=0.010^{*}, p=0.011^{*}, p=0.012^{*}\right.$, $p=0.016^{*}, p=0.019^{*}, p=0.026^{*}$ ) HbA1c - Glycated Haemoglobin, FBS - Fasting Blood Sugar, BMI - Body Mass Index, WHR - Waist Hip Ratio, SLCT - Six Letter Cancellation Test, GHQ - General Health Questionnaire, PSS - Perceived Stress Scale, STAI - State and Trait Anxiety, IDRS - Indian Diabetes Risk Score, QOL - Quality of Life, SD - Standard Deviation.

\section{Discussion}

Chandigarh is a city having highest per capita income in the country and comprises of a heterogenous population. According to ICMR-India DIABETES [INDIA], a study was done in four different regions of India including Tamil Nadu, Jharkhand, Maharashtra and Chandigarh and they found that the occurrence of Diabetes in Chandigarh was higher in both urban and rural areas in comparison with the other three regions. They further reported that the occurrence of prediabetes was higher in urban areas in three regions except Chandigarh where Prediabetes was more prevalent among rural (14.7\%) localities in comparison with urban (14.5\%) areas. Moreover, based on Indian Diabetes Risk Score (IDRS) in Chandigarh (18.7\%), the subjects were found to have high risk of development of Diabetes. The mean HbA1c was highest in Chandigarh in both urban and rural areas (urban-8.7 and rural-9.3) in comparison with other three regions. (6).

Glycated haemoglobin is the most reliable method for detecting the glycaemic index in the individuals. A longitudinal study carried out for six months at VASK Yoga centre, Bangalore studied the proportion of Diabetic patients who adhered to Yoga and its effects were analysed on their blood glucose parameters at baseline, 3 and 6 months. Interestingly, their findings revealed that around $50 \%$ of Diabetic patients complied to Yoga protocol for a period of six months and benefitted by this programme. Their HbA1c levels reduced by the end of $3^{\text {rd }}$ month and other clinical parameters also showed improvement (34). A meta-analysis has revealed beneficial effects of Yoga which included findings showing that Yoga significantly decreased HbAc1 levels and cholesterol besides exerting significant clinical outcomes (35). Recently, Ramamoorthy et al. have designed a protocol to perform the systematic review and meta-analyses on the role of Yoga on prediabetic people/people who are at high risk of developing Diabetes (36).

The present study shows the efficacy of three months national consensus DYP specially designed for Prediabetics (high risk individuals) and Diabetics on the biochemical, anthropometric and psychological parameters. In our study, we used IDRS to assess the risk of Diabetes and Prediabetes among the rural female population. Further, the high risk ( $>60)$ individuals underwent three months Diabetic Yoga Protocol. The results of our study shows statistically significant changes in Glycated haemoglobin $(p=0.010)$, BMI $(p=0.006)$, IDRS $(p<0.001)$, sustained Attention ( $p=0.012)$, General Health $(p=0.002)$, State anxiety $(p=0.001)$, PSS $(p=0.004)$, social $(p=0.006)$, and psychological $(p=0.026)$, domains of QOL and overall QOL $(\mathrm{p}<0.001$ ) (Shown in table 4 and figure 2: a, b, c, d, e, f, g) in DYP group. Our study showed the beneficial effects of Yoga on high risk individuals. However, no statistically significant changes were seen for Fasting blood sugar, Waist hip ratio, Physical and environmental domain of QOL in DYP Group.

In this study the glycaemic parameters i.e. Glycated haemoglobin was significantly reduced even though FBS values 


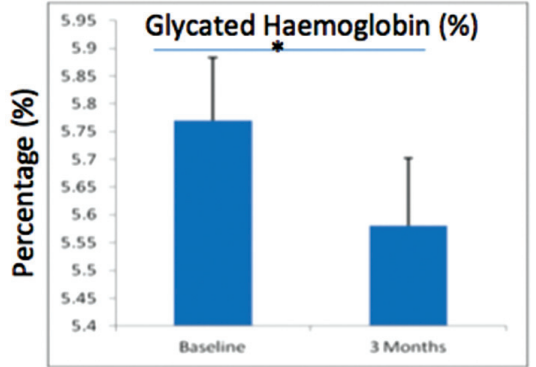

(a)

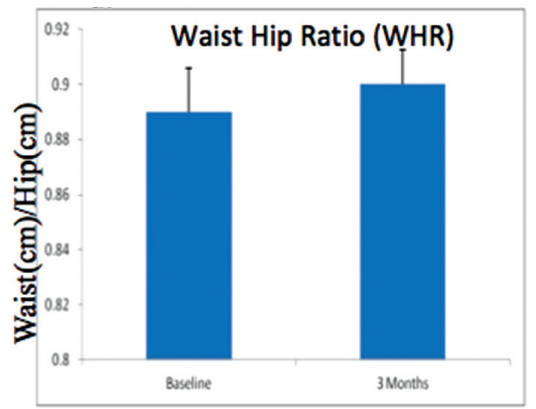

(d)

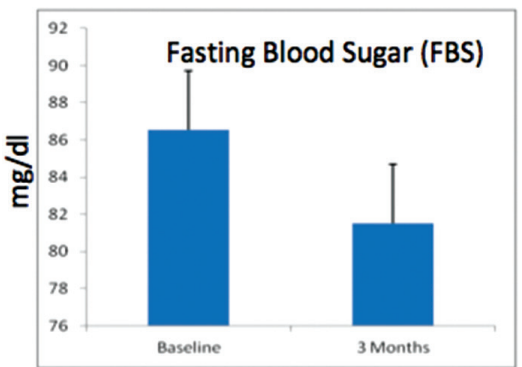

(b)

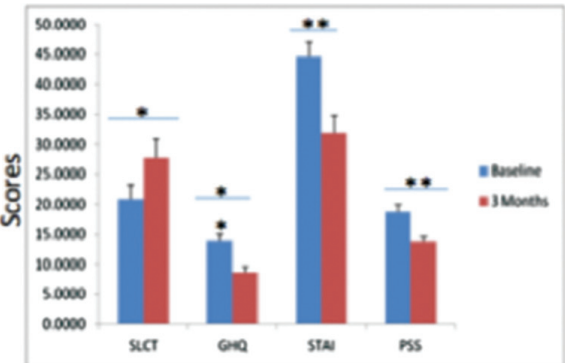

(e)

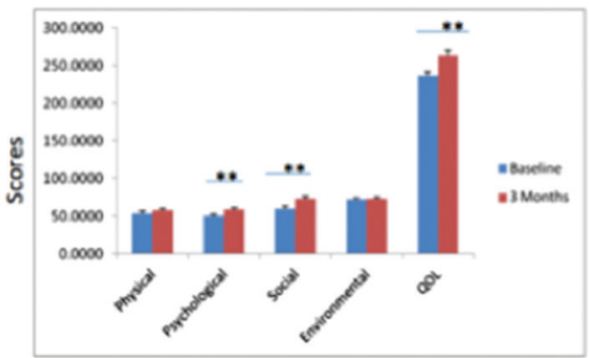

(g)

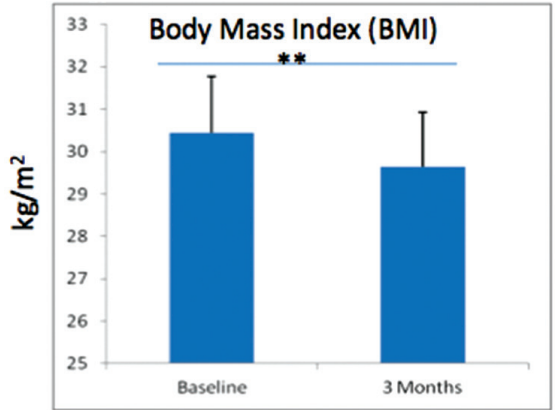

(c)

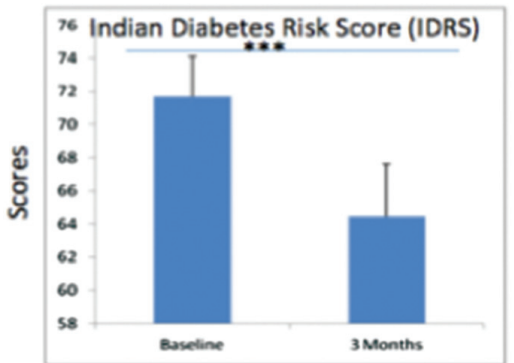

(f)

Fig. 2: Bar Diagrams showing changes for selected parameters i.e. Biochemical (a, b), Anthropometric (c, d), Psychological (e), Risk Status (f and Quality of Life (g) assessments taken at baseline and after 3 months with statistical significance for selected parameters in DYP Group

Data expressed in Bar Diagrams with standard unit values for each variable $\left(p<0.001^{* * *}, p=0.001^{* *}, p=0.002^{* *}, p=0.004^{* *}, p=0.006^{* *}, p=0.010^{*}, p=0.012^{*}\right.$, $p=0.026^{*}$ ) in DYP Group.

did not significantly. However, the decrease in mean FBS change was observed. Our results are consistent with various studies where glycaemic parameters show improvement by Yoga practices. (37-39). However, a study done by Hegde et al. (2013) showed mixed results and found no improvements on HBA1c but improvement in FBS (40). The present study shows significant decrease in BMI among the participants, but no improvement was seen in WHR. Similar results were reported by other studies having improved BMI but no improvements at WHR $(40,37)$. However, none of these studies used a standardized national consensus DYP protocol of Yoga. In contrast, some studies show improvements at WHR after other Yogic practices. $(41,42)$. Based on IDRS, there is a statistically significant difference among the high- risk individuals. A study conducted by Keerthi et al (2009) also shows statistically significant improvement on IDRS in Diabetics and Prediabetics in comparison with controls (44). Sustained attention was also measured in our study. Sustained attention is the ability of an individual to perform the task within specific time, which further shows the individual focus and mental stability. General health includes overall well-being of the individuals, not just freedom from diseases which can be analyzed by a standardized battery. For instance, our study shows improvements in general health and sustained attention (43) of the individuals after Yoga practices. These results are consistent with another study (44). Perceived stress is another parameter, which refers to the individual response to interaction with the environment. In our study, statistically significant improvement was found among Yoga participants on Perceived Stress after Yoga, which was documented, in some another studies $(45,46)$. Additionally, State Anxiety (current feeling of the individual) $(47,48)$ and Overall QOL $(49,50)$ were reportedly improved and these results are consistent with contemporary studies. 


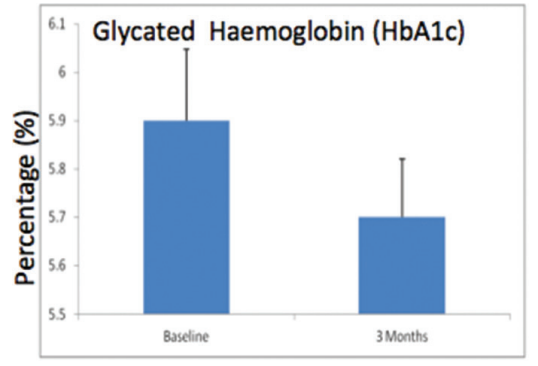

(a)

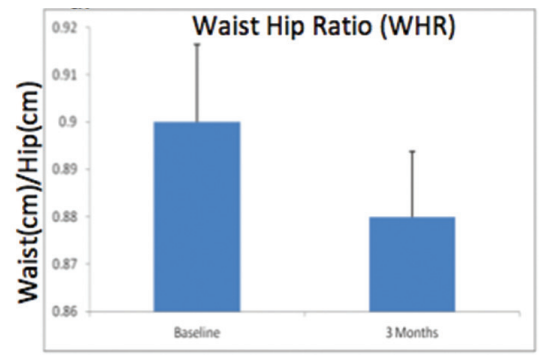

(d)

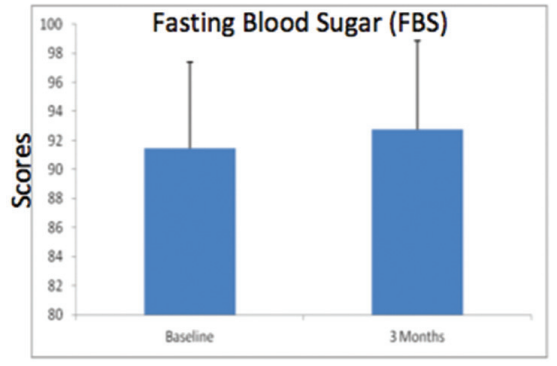

(b)

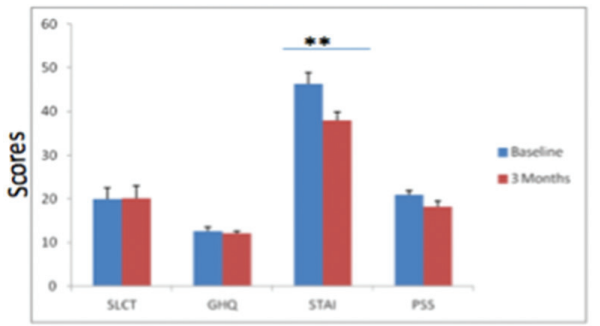

(e)

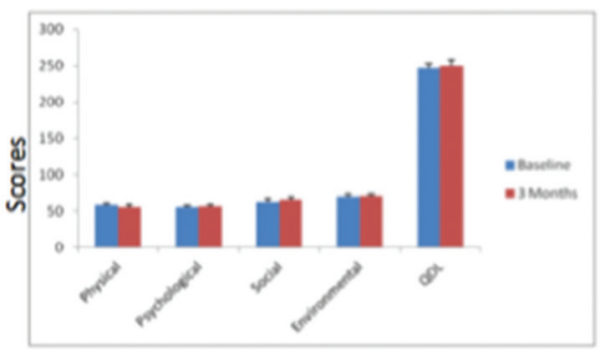

(g)

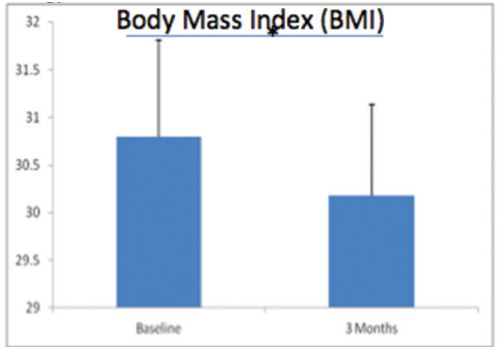

(c)

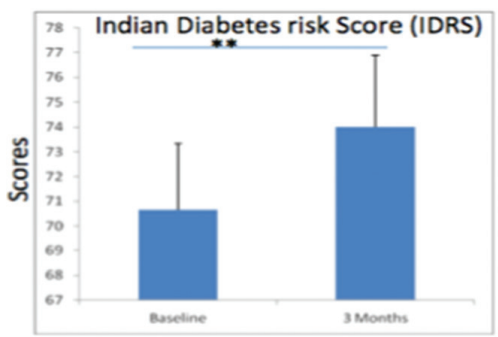

(f)

Fig. 3: Bar Diagrams showing changes for selected parameters i.e Biochemical (a, b), Anthropometric (c, d), Psychological (e), Risk Status (f), and Quality of Life (g) assessments taken at baseline and after 3 months with statistical significance for selected parameters in control Group. Data expressed in Bar Diagrams with standard unit values for each variable $\left(p=0.011^{*}, p=0.016^{*}, p=0.019^{*}\right)$ in Control Group.
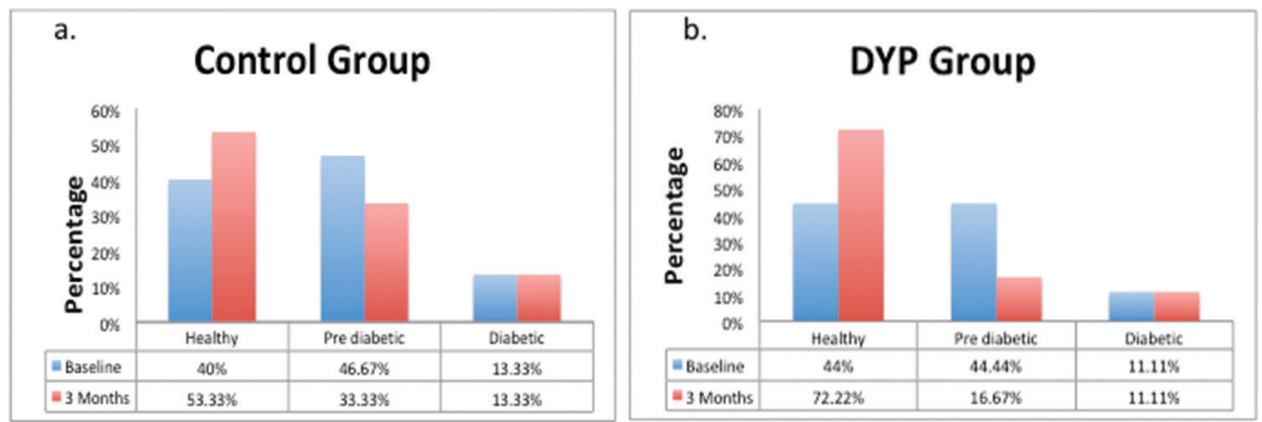

Fig. 4: Conversion rate of prediabetics and newly Diabetics in DYP and Control group after 3 months.

In control group, IDRS was increased significantly and BMI and STAI was found reduced significantly after three months, as shown in Figure 3 and Table 4. However, no significant changes were observed in the level of HbA1c, FBS, WHR, Sustained Attention, Perceived stress, Physical, Psychological and Social measures. Environmental domain and Overall QOL in controls, in comparison with DYP group, has been shown in Figure 3 (a, b, c, d, e, f, g) and Table 4. Both the groups shows significant decline in BMI (DYP-p < .006, Control- $\mathrm{p}<.011$ ). In control group the decrease in BMI was observed which may be due to dietary pattern of the control group. The limitation of the study that it did not control the dietary pattern of the study 
participants remain to be recapitalised by undertaking controlled studies such as barracks, boarding schools or prisons. Moreover, both the groups also showed significant decline in State Anxiety (DYP- $p=0.001$, Control- $p=0.019^{*}$ ). The reason for decline in State Anxiety in Control Group was unclear. However, other Psychological parameters in control group did not shown improvements in comparison with DYP Group.

The breathing techniques focus upon conscious slow breathing and long exhalation, which is believed to trigger parasympathetic nervous system thus bringing positive changes. Diabetes mellitus is linked with various psychological factors like stress and anxiety. Stress hampers immune system of the body and has a poor impact on psychological state of an individual. Yoga is believed to increase the insulin sensitivity of beta cells (pancreas) to glucose signals in Diabetes mellitus. It is possible that Yoga, through neuro-endocrinal system, modifies the insulin secretion by unknown mechanisms, enabling maintenance of the normal glucose level in the body besides alleviating insulin resistance.

\section{Limitations and scope for future study}

The present study is the pilot study carried out on the $33 \mathrm{fe}-$ male participants. This study lacked the male participants and the sample size was small. Due its small sample size, we are unable to generalize its impact on larger population. However, the pilot study suggests that Yoga not only exerts effective control of glycaemic and anthropometric parameters but also the psychological parameters among high risk individuals.

\section{Conclusion}

The results from our study show the beneficial effect of Yoga especially when encapsulated as DYP. Therefore, this study supports the efficacy of DYP on glycaemic parameters on high risk females. Yoga reduces the risk of Diabetes among high risk individuals by reducing the IDRS score among high risk females. It is difficult to conclude whether the amelioration of physical and biochemical parameters result from the psychological changes brought about by Yoga as it will require long term studies.

\section{Acknowledgements}

The authors acknowledge the MHRD/ICSSR for supporting this research. The authors acknowledge Ms. Sarika Dhiman, Dr Davinder Dhawan, and Bhai Ghaniya ji (BGJ) institute of health, Panjab University, Chandiagarh for helping in the study. The authors would like to thank the all the study participants for their involvement in the trial.

\section{Authors contribution}

NK: Writing, Review, Study Design and Analysis of the Data.

NM: Conceptualization and editing of Manuscript

DM: Writing of the manuscript.

SP: Writing, validation of data

RM: Writing, co-conceptualization, editing of the manuscript. SR: Validation of data.

\section{Source of funding}

This study has been supported by University Grants Commission (UGC) via Letter No. 15-9(June 2014)/2014(NET).

\section{Ethical statement}

University Institutional Ethics Committee (PUIEC) vide letter no. PUIEC/2017/80/A-1/08/08.

\section{Conflict of interest}

The authors declare no conflict of interest.

Received Date: 03-04-20; Revised Date: 31-05-20

Accepted Date: $12-06-20$

\section{References}

1. Dall TM, Zhang Y, Chen YJ, Quick WW, Yang WG, Fogli J. The economic burden of diabetes. Health affairs. 2010 Feb 1;29(2):297-303.

2. Dall TM, Yang W, Halder P, Pang B, Massoudi M, Wintfeld N, Semilla AP, Franz J, Hogan PF. The economic burden of elevated blood glucose levels in 2012: diagnosed and undiagnosed diabetes, gestational diabetes mellitus, and prediabetes. Diabetes care. 2014 Dec 1;37(12):3172-9. Edition ID.

3. International Diabetes Federation. IDF Diabetes Atlas, 8th edn. Brussels, Belgium: International Diabetes Federation, 2017.

4. Chawla A, Chawla R, Jaggi S. Microvasular and macrovascular complications in diabetes mellitus: distinct or continuum?. Indian journal of endocrinology and metabolism. 2016 Jul;20(4):546.

5. Mohan V, Deepa M, Anjana RM, Lanthorn H, Deepa R. Incidence of diabetes and pre-diabetes in a selected urban south Indian population (CUPS-19). Journal of Association of Physicians of India. 2008 Mar 1;56(3):152-7.

6. Anjana RM, Pradeepa R, Deepa M, Datta M, Sudha V, Unnikrishnan R, Bhansali A, Joshi SR, Joshi PP, Yajnik CS, Dhandhania VK. Prevalence of diabetes and prediabetes (impaired fasting glucose and/or impaired glucose tolerance) in urban and rural India: Phase I results of the Indian Council of Medical Research-INdia DIABetes (ICMR-INDIAB) study. Diabetologia. 2011 Dec 1;54(12):3022-7.

7. Anjana RM, Rani CS, Deepa M, Pradeepa R, Sudha V, Nair HD, Lakshmipriya N, Subhashini S, Binu VS, Unnikrishnan R, Mohan V. Incidence of diabetes and prediabetes and predictors of progression among Asian Indians: 10-year follow-up of the Chennai Urban Rural Epidemiology Study (CURES). Diabetes care. 2015 Aug 1;38(8):1441-8.

8. American Diabetes Association. Report of the expert committee on the diagnosis and classification of diabetes mellitus. Diabetes care. 2003 Jan 1;26(suppl 1):s5-20.

9. Neel JV. Diabetes mellitus: a "thrifty" genotype rendered detrimental by "progress"?. American journal of human genetics. 1962 Dec;14(4):353.

10. Safford MM, Russell L, Suh DC, Roman S, Pogach L. How much time do patients with diabetes spend on self-care?. The Journal of the American Board of Family Practice. 2005 Jul 1;18(4):262-70.

11. Mohan V, Deepa R, Deepa M, Somannavar S, Datta M. A simplified Indian Diabetes Risk Score for screening for undiagnosed diabetic subjects. The Journal of the Association of Physicians of India. 2005 Sep 1;53:759-63.

12. Hamman RF. Genetic and environmental determinants of non-insulindependent diabetes mellitus (NIDDM). Diabetes/metabolism reviews. 1992 Dec;8(4):287-338.

13. Manson JE, Stampfer MJ, Colditz GA, Willett WC, Rosner B, Hennekens CH, Speizer FE, Rimm EB, Krolewski AS. Physical activity and incidence of non-insulin-dependent diabetes mellitus in women. The Lancet. 1991 Sep 28;338(8770):774-8.

14. Bali, H. K. (2013). Yoga-an ancient solution to a modern epidemic. Ready for prime time?. Indian heart journal, 65(2), 132-136. 
15. Gupta N, Khera S, Vempati RP, Sharma R, Bijlani RL. Effect of yoga based lifestyle intervention on state and trait anxiety. Indian journal of physiology and pharmacology. 2006 Jan 21;50(1):41.

16. Laaksonen DE, Lindström J, Lakka TA, Eriksson JG, Niskanen L, Wikström K, Aunola S, Keinänen-Kiukaanniemi S, Laakso M, Valle TT, Ilanne-Parikka P. Physical activity in the prevention of type 2 diabetes: the Finnish diabetes prevention study. Diabetes. 2005 Jan 1;54(1):158-65.

17. Lindström J, Ilanne-Parikka P, Peltonen M, Aunola S, Eriksson JG, Hemiö K, Hämäläinen $H$, Härkönen $P$, Keinänen-Kiukaanniemi S, Laakso $M$, Louheranta A. Sustained reduction in the incidence of type 2 diabetes by lifestyle intervention: follow-up of the Finnish Diabetes Prevention Study. The Lancet. 2006 Nov 11;368(9548):1673-9.

18. Nayak NN, Shankar K. Yoga: a therapeutic approach. Physical Medicine and Rehabilitation Clinics. 2004 Nov 1;15(4):783-98.

19. Shiju R, Thomas D, Al Arouj M, Sharma P, Tuomilehto J, Bennakhi A. Effect of Sudarshan Kriya Yoga on anxiety, depression, and quality of life in people with type 2 diabetes: A pilot study in Kuwait. Diabetes \& Metabolic Syndrome: Clinical Research \& Reviews. 2019 May 1;13(3):1995-9.

20. Jyotsna VP. Prediabetes and type 2 diabetes mellitus: Evidence for effect of yoga. Indian journal of endocrinology and metabolism. 2014 Nov;18(6):745.

21. Eriksson KF, Lindgärde F. Prevention of Type 2 (non-insulin-dependent) diabetes mellitus by diet and physical exercise The 6-year Malmö feasibility study. Diabetologia. 1991 Dec 1;34(12):891-8.

22. Tuomilehto J, Lindström J, Eriksson JG, Valle TT, Hämäläinen H, IlanneParikka P, Keinänen-Kiukaanniemi S, Laakso M, Louheranta A, Rastas M, Salminen V. Prevention of type 2 diabetes mellitus by changes in lifestyle among subjects with impaired glucose tolerance. New England Journal of Medicine. 2001 May 3;344(18):1343-50.

23. Li G, Zhang P, Wang J, Gregg EW, Yang W, Gong Q, Li H, Li H, Jiang Y, An Y, Shuai Y. The long-term effect of lifestyle interventions to prevent diabetes in the China Da Qing Diabetes Prevention Study: a 20-year follow-up study. The Lancet. 2008 May 24;371(9626):1783-9.

24. Echouffo-Tcheugui JB, Dagogo-Jack S. Preventing diabetes mellitus in developing countries. Nature Reviews Endocrinology. 2012 Sep;8(9):557.

25. Knowler WC, Barrett-Connor E, Fowler SE, Hamman RF, Lachin JM, Walker EA, Nathan DM. Reduction in the incidence of type 2 diabetes with lifestyle intervention or metformin. The New England journal of medicine. 2002 Feb;346(6):393-403.

26. Ramachandran A, Snehalatha C, Mary S, Mukesh B, Bhaskar AD, Vijay V. The Indian Diabetes Prevention Programme shows that lifestyle modification and metformin prevent type 2 diabetes in Asian Indian subjects with impaired glucose tolerance (IDPP-1). Diabetologia. 2006 Feb 1;49(2):289-97.

27. Arumugam G, Nagarathna R, Majumdar V, Singh M, Srinivasalu R, Sanjival R, Ram VS, Nagendra HR. Yoga-based lifestyle treatment and composite treatment goals in type 2 Diabetes in a rural South Indian setup-a retrospective study. Scientific reports. 2020 Apr 14;10(1):1-0.

28. Natu MV, Agarwal AK. Testing of stimulant effects of coffee on the psychomotor performance: An exercise in clinical pharmacology. Indian journal of pharmacology. 1997 Jan 1;29(1):11.

29. Spielberger CD, Gorsuch RL. State-trait anxiety inventory for adults: Manual and sample: Manual, instrument and scoring guide. Consulting Psychologists Press; 1983.

30. Cohen S, Kamarck T, Mermelstein R. Perceived stress scale. Measuring stress: a guide for health and social scientists. 1994.

31. Goldberg, D.P., Gater, R., Sartorius, N., Ustun, T.B., Piccinelli, M., Gureje, O. and Rutter, C., 1997. The validity of two versions of the GHQ in the WHO study of mental illness in general health care. Psychological medicine, 27(1), pp.191-197.

32. World Health Organization. The world health organization quality of life (WHOQOL)-BREF. World Health Organization; 2004.

33. Singh AK, Kaur N, Kaushal S, Tyagi R, Mathur D, Sivapuram MS, Metri K, Bammidi S, Podder V, Modgil S, Khosla R. Partitioning of radiological, stress and biochemical changes in pre-diabetic women subjected to
Diabetic Yoga Protocol. Diabetes \& Metabolic Syndrome: Clinical Research \& Reviews. 2019 Jul 1;13(4):2705-13.

34. Angadi P, Jagannathan A, Thulasi A, Kumar V, Umamaheshwar K, Raghuram $\mathrm{N}$. Adherence to yoga and its resultant effects on blood glucose in type 2 diabetes: a community-based follow-up study. International journal of yoga. 2017 Jan;10(1):29.

35. Cui J, Yan JH, Yan LM, Pan L, Le JJ, Guo YZ. Effects of yoga in adults with type 2 diabetes mellitus: A meta analysis. Journal of diabetes investigation. 2017 Mar;8(2):201-9.

36. Ramamoorthi R, Gahreman D, Moss S, Skinner T. The effectiveness of yoga to prevent diabetes mellitus type 2: A protocol for systematic review and meta-analysis. Medicine. 2019 Jan;98(3).

37. Hegde SV, Adhikari P, Kotian S, Pinto VJ, D'Souza S, D'Souza V. Effect of 3-month yoga on oxidative stress in type 2 diabetes with or without complications: a controlled clinical trial. Diabetes care. 2011 Oct 1;34(10):2208-10.

38. Kacker S, Saboo N, Sharma S, Sorout J. Quasi prospective comparative study on effect of yoga among prediabetics on progression of cardiovascular risk factors. International journal of yoga. 2019 May;12(2):114.

39. Singh S, Malhotra V, Singh KP, Sharma SB, Madhu SV, Tandon OP. A preliminary report on the role of yoga asanas on oxidative stress in noninsulin dependent diabetes mellitus. Indian Journal of Clinical Biochemistry. 2001 Jul 1;16(2):216-20.

40. Hegde SV, Adhikari P, Shetty S, Manjrekar P, D'Souza V. Effect of communitybased yoga intervention on oxidative stress and glycemic parameters in prediabetes: a randomized controlled trial. Complementary therapies in medicine. 2013 Dec 1;21(6):571-6.

41. Tundwala V, Gupta RP, Kumar S, Singh VB, Sandeep BR, Dayal P, Prakash P. A study on effect of yoga and various asanas on obesity, hypertension and dyslipidemia. Int J Basic Appl Med Sci. 2012;2(1):93-8.

42. Keerthi GS, Pal P, Pal GK, Sahoo JP, Sridhar MG, Balachander J. Effect of 12 weeks of yoga therapy on quality of life and Indian diabetes risk score in normotensive Indian young adult prediabetics and diabetics: randomized control trial. Journal of clinical and diagnostic research: JCDR. 2017 Sep;11(9):CC10.

43. Kumar S, Telles S. Meditative states based on yoga texts and their effects on performance of a letter-cancellation task. Perceptual and Motor Skills. 2009 Dec;109(3):679-89.

44. Khemka SS, Ramarao NH, Hankey A. Effect of integral yoga on psychological and health variables and their correlations. International journal of yoga. 2011 Jul;4(2):93.

45. Hewett ZL, Pumpa KL, Smith CA, Fahey PP, Cheema BS. Effect of a 16-week Bikram yoga program on perceived stress, self-efficacy and healthrelated quality of life in stressed and sedentary adults: A randomised controlled trial. Journal of science and medicine in sport. $2018 \mathrm{Apr}$ 1;21(4):352-7.

46. Sharma VK, Trakroo M, Subramaniam V, Rajajeyakumar M, Bhavanani AB, Sahai A. Effect of fast and slow pranayama on perceived stress and cardiovascular parameters in young health-care students. International journal of yoga. 2013 Jul;6(2):104.

47. Satyapriya M, Nagarathna R, Padmalatha V, Nagendra HR. Effect of integrated yoga on anxiety, depression \& well being in normal pregnancy. Complementary therapies in clinical practice. 2013 Nov 1;19(4):230-6.

48. Gupta N, Khera S, Vempati RP, Sharma R, Bijlani RL. Effect of yoga based lifestyle intervention on state and trait anxiety. Indian journal of physiology and pharmacology. 2006 Jan 21;50(1):41.

49. Jyotsna VP, Joshi A, Ambekar S, Kumar N, Dhawan A, Sreenivas V. Comprehensive yogic breathing program improves quality of life in patients with diabetes. Indian journal of endocrinology and metabolism. 2012 May;16(3):423.

50. Shiju R, Thomas D, Al Arouj M, Sharma P, Tuomilehto J, Bennakhi A. Effect of Sudarshan Kriya Yoga on anxiety, depression, and quality of life in people with type 2 diabetes: A pilot study in Kuwait. Diabetes \& Metabolic Syndrome: Clinical Research \& Reviews. 2019 May 1; 13(3):1995-9. 